\title{
Daten-Ökosysteme wider Willen: Herausforderungen des Pay-as-you-live-Geschäftsmodells im Kontext deutscher Krankenversicherungen
}

\author{
Nele Wulf • Stefanie Betz
}

Eingegangen: 5. Januar 2021 / Angenommen: 12. März 2021 / Online publiziert: 1. April 2021

(C) Der/die Autor(en) 2021

Zusammenfassung In dem vorliegenden Beitrag wird der aktuelle Trend, Payas-you-live-Systeme (PAYL) in Verbindung mit Krankenversicherungen anzubieten, analysiert. PAYL-Systeme bedeutet konkret die kontinuierliche Erfassung von Gesundheitsdaten mithilfe technischer Geräte und Übermittlung dieser Daten an Versicherungen sowie die Auszahlung von Boni für erreichte Fitnessziele. Zunächst wird eine Definition von PAYL-Systemen dargelegt, dann werden die technischen Rahmenbedingungen erläutert, schließlich werden die Auswirkungen dieser soziotechnischen Systeme näher beleuchtet. Dies geschieht auf Grundlage unserer empirischen Untersuchung. Die drei identifizierten Hauptkonflikte betreffen die Genese von PAYL, seine Auswirkungen auf den Wert der Datensouveränität besonders für Versicherte und die Schwierigkeit, für den Anspruch auf Prävention und Kostenersparnis durch PAYL Evidenz zu erzeugen. Diese Konflikte werden diskutiert, um sowohl die direkten wie auch indirekten Auswirkungen der Digitalisierung und der Ökonomisierung des Sozialen durch PAYL zu beleuchten.

Schlüsselwörter Pay-as-you-live $\cdot$ Sozio-technische Systeme $\cdot$ Digitale Souveränität · Krankenversicherungen · Selbstvermessung · Daten-Ökosystem

\footnotetext{
N. Wulf $(\bowtie) \cdot$ S. Betz

Fakultät Informatik, Hochschule Furtwangen I Furtwangen University, Furtwangen, Deutschland E-Mail: wun@hs-furtwangen.de

S. Betz

E-Mail: besi@hs-furtwangen.de
} 


\title{
Unintended Data ecosystems: Challenges of the Pay-as-you-live Business Model in the Context of German Health Insurers
}

\begin{abstract}
This paper analyzes the current trend of offering pay as you live systems in conjunction with health insurance. In concrete terms, PAYL-systems mean the continuous recording of health data with the aid of technical devices and transmission of this data to insurance companies, as well as the payment of bonuses for fitness goals achieved. First, a definition of PAYL-systems is presented, then the technical framework is explained, and finally the effects of these socio-technical systems are examined in more detail. This is done on the basis of our empirical investigation. The three main conflicts identified relate to the genesis of PAYL, its impact on the value of data sovereignty especially for insured individuals, and the difficulty of generating evidence for the PAYL prevention and cost savings claim. These conflicts are discussed in order to shed light on both the direct and indirect effects of digitization and the economization of the social through PAYL.
\end{abstract}

Keywords Pay-as-you-live $\cdot$ Socio-technical systems $\cdot$ Digital sovereignty $\cdot$ Health insurance $\cdot$ Self-tracking $\cdot$ System of Systems

\section{Einleitung}

10.000 Schritte pro Tag ist eins der populärsten Fitnessziele unsere Zeit und kaum jemand zählt diese ohne technische Hilfsmittel. Doch Fitness-Tracker, Smartwatches und Gesundheits-Apps werden nicht mehr nur zur Messung der gesundheitlichen Leistung sowie zur Selbstoptimierung eingesetzt. Zunehmend vermessen sich Menschen digital und geben diese privaten, körperbezogenen Daten freiwillig an Dritte weiter, zum Beispiel an Krankenversicherungen. Diese wiederum bieten Bonusprogramme und Pay-as-you-live-Tarife (PAYL) an, mit denen sie einen gesundheitsbewussten bzw. präventiven Lebensstil monetär oder mit anderen Leistungen belohnen wollen. Vor diesem Hintergrund erscheinen PAYL-Systeme als eine Signatur der Gegenwartsgesellschaft: Digitalisierung einerseits und die Ökonomisierung des Sozialen andererseits. Es gilt daher, eine Ökosystem-Perspektive an PAYL-Systeme anzulegen, also diese inklusive des Zusammenspiels mit anderen (datengetriebenen) Geschäftsmodellen und (technischen) Systemen zu verstehen.

Der Beitrag geht der Frage nach, wie sozio-technische PAYL-Systeme von Krankenkassen aussehen, wie sie verwendet werden und welche (Haupt-)Konflikte um die Implementierung als (datengetriebenes) Geschäftsmodell entstehen bzw. entstehen könnten. Das sozio-technische System PAYL wird zur Illustration anhand der AOK Plus vorgestellt, da es eines der ausgereiftesten Angebote auf dem deutschen Markt darstellt und die technische Umsetzung den momentanen Standardfall darstellt.

Der Beitrag strukturiert sich folgendermaßen: Zunächst wird eine Definition von PAYL-Systemen gegeben, dann werden die technischen Komponenten eines solchen Systems erläutert sowie der aktuelle Stand auf dem Markt kurz vorgestellt. Anschließend wird unsere Interviewstudie erläutert, deren Ergebnisse dann diskutiert werden, um sowohl direkte als auch indirekte Auswirkungen der Digitalisierung 
und der Ökonomisierung des Sozialen beleuchten zu können. Abschließend erfolgen die Zusammenfassung und der Ausblick.

\section{Einführung in PAYL Systeme}

Da das Thema relativ neu ist und die wissenschaftliche Betrachtung noch am Anfang steht, wird zunächst unsere Definition von PAYL vorgestellt. Um ein Programm als genuin PAYL zu bezeichnen, müssen die folgenden fünf Kriterien erfüllt sein: (1) Bei der Vermessung werden Körperdaten ohne eigenes Zutun (Selbsteingabe) erhoben. (2) Die Vermessung erfolgt digital mithilfe entsprechender Hard- und Software, ist also auf ein Informations- und Kommunikationssystem angewiesen. (3) Die Versicherungen legen in einer Zielvereinbarung bspw. Schwellwerte fest, ab denen ein Fitnessziel als erreicht gilt und ein Bonus ausgezahlt wird. (4) Anders als beim klassischen Bonusheft, wo auch ad hoc Gesundheitsdaten erhoben werden (z. B. Vorsorgeuntersuchungen), bedarf die Erhebung der Gesundheitsdaten Kontinuität. (5) Die Belohnung kann vielgestaltig sein und beispielsweise Geldzahlungen, Tarifreduzierungen, Rabattierungen bei Drittanbietern, Einkaufsgutscheine und Zuzahlungen zu Fitnessgeräten umfassen.

PAYL-Tarife gibt es - im Gegensatz zu PAYL-Bonusprogrammen für Krankenversicherungen - in Deutschland bislang noch nicht. Einzig die Generali bietet PAYLTarife in Zusammenarbeit mit Vitality seit 2016 für spezielle Berufs- und Risikoversicherungen an und will Krankenversicherungen folgen lassen. Bonusprogramme, die PAYL-Aspekte beinhalten, sind bislang nur im Bereich der gesetzlichen Krankenversicherungen vorhanden. Fünf der elf nach Mitgliederzahl größten gesetzlichen Krankenversicherungen (GKV) bieten Varianten von PAYL an.

\subsection{Technologisches System PAYL}

Die technische Dimension der PAYL-Programme (siehe Abb. 1) umfasst zunächst die Tracking-Technologien. Wearables sind mit diversen Sensoren ausgestattet, über die Vitalzeichen und Trainingsleistungen der Tragenden erfasst und zur Weiterverarbeitung an ein leistungsfähiges mobiles Gerät wie zum Beispiel ein Smartphone übertragen werden (vgl. Kruse Brandão und Wolfram 2018). Die Verbindung zwischen den beiden Geräten findet in der Regel über Bluetooth Low Energy (BLE) statt. Um die gesammelten Daten an den Versicherer weiterzugeben, kommen Gesundheits-Apps zum Einsatz. Gesundheits-Apps werden von Krankenversicherungen verwendet, um ihren Versicherten Präventions- und Bonusprogramme sowie Informationen und bürokratische Dienste anbieten zu können (Evers-Wölk et al. 2018).

Nach aktuellem Kenntnisstand lässt sich keine Anwendung einer Krankenversicherung direkt mit dem Wearable verbinden. Daher ist es, um von PAYL-basierten Bonusprogrammen profitieren zu können, nicht nur erforderlich, die notwendigen Geräte zu besitzen, sondern auch, die damit kompatiblen Gesundheitsplattformen zu nutzen. Das setzt ein Nutzungskonto bei der jeweiligen Plattform voraus. Dafür wird ein aktuelles Smartphone mit Apples iOS oder Googles Android Betriebssystem sowie ein kompatibles Wearable benötigt. Letzteres wird bei der Auswahl nur 


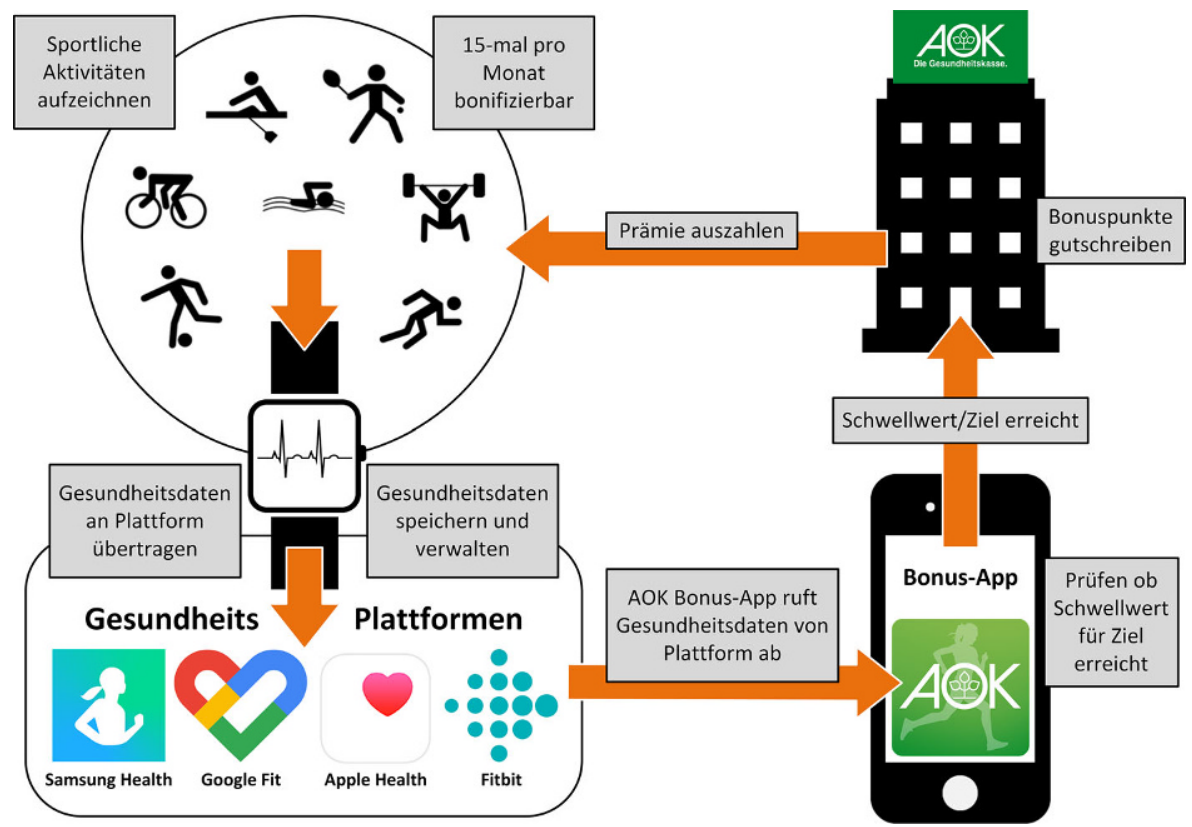

Abb. 1 Systemkomponenten und Datenfluss am Beispiel der AOK Plus

noch vom Funktionsumfang der verbauten Sensoren bestimmt. Je nachdem, welche sportliche Leistung ein Bonusprogramm mit einem Bonus gegenrechnet, müssen Sensoren vorhanden sein, um die entsprechenden Daten erfassen zu können. Die installierte Gesundheitsplattform verbindet sich mit dem Wearable und stellt per Programmierschnittstelle (API: Application Programming Interface) die erfassten Sensordaten den Gesundheits-Apps zur Verfügung. Das ermöglicht eine Validierung der festgelegten Fitnessziele anhand der durch die Plattformen bereitgestellten Daten.

Die gewählte Plattform ist auch ausschlaggebend für den Speicherort der erfassten Gesundheitsdaten. So ist bei Apple eine Speicherung ausschließlich auf dem Gerät möglich. Samsung agiert freizügiger und lässt die Wahl, ob die Datensynchronisation zwischen Gerätespeicher und Cloud-Server stattfinden soll. Da Google seine Plattform weniger an das eigene Betriebssystem koppelt, sondern sie mehr als reinen Onlinedienst versteht, ist es hier kaum möglich, die Kontrolle zu behalten und den Datenabfluss lokal gespeicherter Gesundheitsdaten einzudämmen. Gesetzlich sind den Krankenversicherungen enge Grenzen gesetzt, welche Daten sie zu welchen Zwecken erheben dürfen. Daher gestalten sie ihre Seite des technischen Systems PAYL eher datensparsam. Sie fragen beispielsweise nur ab, ob der Schwellwert für ein von ihnen festgelegtes Fitnessziel erreicht wurde, aber nicht wie viele Schritte dafür genau gelaufen worden sind. Allerdings werden, wie beschrieben, diese Daten zwar der Krankenkasse nicht zugespielt, nichtsdestotrotz aber erhoben und gespeichert. PAYL-Systeme, wie sie aktuell in Deutschland realisiert werden, sind auf Plattformanbieter wie Google angewiesen und im Moment zumindest nicht gewillt, 
Tab. 1 Sampleübersicht

\begin{tabular}{ll}
\hline Interviewkategorie & Anzahl der Interviews \\
\hline Gesetzliche Krankenversicherung (GKV) & 7 \\
Private Krankenversicherung (PKV) & 3 \\
Forschende mit primär wissenschaftlichem Blick auf PAYL & 5 \\
Softwareentwicklungsfirmen, die mit Krankenkassen zusammenarbeiten & 2 \\
Beratungseinrichtungen mit öffentlicher Förderung & 2 \\
Sozialverbände/Patientenverbände & 2 \\
Hersteller von Tracking-Geräten & 2 \\
Quantified-Self-Expert*innen/Aktivist*innen & 2 \\
Rechtsaufsicht & 1 \\
Aktuarvereinigung & 1 \\
Landesärztekammer & 1 \\
Scoring-Unternehmen & 1 \\
Verbraucherschutz & 1 \\
Anwender*innen & 9 \\
\hline
\end{tabular}

eigene Infrastruktur aufzubauen. Wenn also Kund*innen an einem PAYL-System teilnehmen wollen, müssen sie der Datenerhebung und Speicherung von Drittanbietern zustimmen. Die dabei von ihnen gesetzten Häkchen (Opt-out) ermöglichen daher, dass Daten in Umlauf geraten, auf die unterschiedlichste Stakeholder Zugriff haben, die ihre jeweils eigenen datengetriebenen Geschäftsmodelle und damit verbundenen Verwendungszwecke verfolgen.

\subsection{Empirische Studie}

Grundlage der vorliegenden Fallstudie sind 29 Interviews mit Expert*innen vorrangig aus dem deutschen Gesundheitswesen, neun Interviews mit Teilnehmenden an PAYL-Bonusprogrammen, eine Gruppendiskussion mit Bio-Hackern sowie eine Dokumentenanalyse. Die Auswahl der Expert*innen wurde im Schneeballsystem durchgeführt und durch ein Theoretical Sampling der PAYL-relevanten Stakeholdern im Gesundheitssystem ergänzt. Alle interviewten Expert*innen verfügen über mehrjährige Erfahrung in ihrem Feld (Tab. 1).

Alle Interviews wurden mithilfe eines semi-strukturierten Leitfadens erhoben, der nach einem Pretest entsprechend angepasst wurde. Die Expert*innen wurden zu folgenden Themenbereichen gefragt: Ihr Bezug zu PAYL, das Verhältnis von Versicherung und Versicherten, Nutzen und Nutzungsbedingungen der Technik, Chancen und Risiken der Technologie, der medizinische Nutzen sowie die Zukunft von PAYL. Bei der Befragung der Anwender*innen und der Bio-Hacker wurden diese Bereiche ebenfalls abgefragt, aber mit einem stärker narrativen Fokus auf die eigenen Erfahrungen und Wünsche bezüglich PAYL. Die durchschnittliche Dauer der Interviews betrug zwischen 45 und $60 \mathrm{~min}$.

Für die Analyse wurde ein Methodenmix verwendet, der die Grounded Theory nach Corbin und Strauss (2015) um Ansätze von Clarke (2005) zur visuellen Analyse von Diskursen ergänzt. Die vollständig transkribierten Interviews wurden von 
der Erstautorin offen codiert, die Codes ergaben sich dabei fast ausschließlich aus dem Material selbst (In-vivo-Codes). Die Gliederung des Code-Baumes spiegelt die Themenbereiche des Fragebogens wider, zudem wurden die im Kontext von PAYL thematisierten Akteure und ihre jeweilige Bewertung und Motivation separat codiert. Der am umfangreichsten codierte Bereich ist der zu den Zukunftsvisionen bezüglich PAYL. Er untergliedert sich in Aussagen zu Herausforderungen, Chancen, Risiken, den Status quo, mögliche Alternativen zu PAYL sowie Aussagen zu angenommenen zukünftigen Verhinderung oder Durchsetzung von PAYL. Zu allen Interviews wurden Memos erstellt, welche den Inhalt kondensiert zusammenfassen sowie gedanklichen Verbindungen zu bereits Analysiertem festhalten. Ausgehend von Dahrendorfs Konflikttheorie (1961) wurden die Aussagen dann entweder der Kategorie Interessenskonflikt (3.1), Wertekonflikt (3.2) oder Wissenskonflikt (3.2) zugeordnet. Die in Kap. 3 vertieften Konflikte lassen sich jeweils einer dieser Konfliktarten zuordnen und sind als Kristallisationspunkte der Debatte im Zusammenhang mit datengetriebenen PAYL-Geschäftsmodellen im Gesundheitswesen zu werten. Um die Interrater-Reliabilität der Ergebnisse zu gewährleisten, wurde die Analyse von zwei weiteren Wissenschaftler*innen iterativ validiert, indem die jeweiligen Schritte und Ergebnisse (Codierung, Memos, Konfliktanalyse) diskutiert und analysiert wurden.

\section{PAYL-Systeme im Kontext eines datengetriebenen Geschäftsmodells}

$\mathrm{Zu}$ beachten gilt, dass den Krankenkassen durch die gesetzlichen Regulierungen, die Versicherungspflicht und die Prinzipien der Solidarität (GKV) bzw. der Äquivalenz (PKV) enge Grenzen in Bezug auf ihr Geschäftsmodell gesetzt sind. Dennoch wandeln die Krankenkassen ihr Geschäftsmodell gerade vom administrierenden, analogen Kostenerstatter zum ganzheitlichen, individualisierten und digitalisierten (und damit datengetriebenen) Gesundheitsdienstleister, der Kundenservice, Prävention, Diagnose und Behandlung (digital) unterstützt (MSC 2018; BCG 2017). Hier setzen auch PAYL-Systeme an. Sie sind dem Bereich des Kundenservice und der Prävention zuzuordnen.

Allerdings sind im Zusammenhang mit PAYL Systemen und datengetriebenen Geschäftsmodellen nicht nur die Krankenkassen selbst zu betrachten. Die Akteure innerhalb von PAYL-Systemen lassen sich basierend auf Wiener et al. (2020) den drei datengetriebenen Geschäftsmodelltypen zuordnen: Die Versicherungen (GKV, PKV) sind dem Data-User-Modell zuzuordnen, die Dienstleister als zusätzliche Akteure dem Data-Facilitator-Modell und die Plattformen dem Data-Supplier-Modell. Die Dienstleister als Data-Facilitator sind aber nur zusätzliche Akutere, kommen in den aktuellen PAYL-Umsetzungen nicht vor und sind nur der Vollständigkeit halber erwähnt. Daher werden sie im Folgenden nicht weiter diskutiert. Wenn ein datengetriebenes Geschäftsmodell verfolgt wird, kann dies zu Konflikten führen, beispielsweise im Bereich der Privatsphäre (Wiener et al. 2020). Ausgehend von der Interviewstudie wurden drei Konfliktbereiche identifiziert, die besondere Hindernisse für eine weitergehende Implementierung von PAYL-Systemen innerhalb von Krankenversicherungen darstellen und im Folgenden diskutiert werden. Die Auswahl ist nicht abschließend, die genannten Probleme sind aber unserer Meinung nach im 
Rahmen einer Diskussion über datengetriebene Geschäftsmodelle elementar, da sie den Entstehungskontext des Modells, seine eigentliche Nutzung und die formulierte Zielvorstellung berühren. Die Genese des Modells ist entscheidend für das Narrativ und dafür, ob die Versicherungen sich zu einem Trend verhalten oder aktiv ein Modell anbieten. Das Moment der Datensouveränität ist im Rahmen eines datengetriebenen Geschäftsmodells besonders aufgrund der Nutzer*innenperspektive von Bedeutung, da aus der Behauptung heraus, dieser Wert könne im System angemessen realisiert werden, die Datenverantwortung auf die einzelnen Versicherten verlagert wird. Die Datensouveränität steht auch in Konflikt mit dem Geschäftsmodell der beteiligten Plattformen (Data-Supplier). Ebenso diskutiert wird die Realisierbarkeit des (auch gesetzlich festgelegten) Ziels des Geschäftsmodells der Krankenkassen als Data-User, Prävention kostenneutral zu gewährleisten.

\subsection{Stichwortgeber Unternehmensberatung}

Unternehmensberatungen scheinen die zentralen Stakeholder zu sein, betrachtet man die Emergenz von PAYL. In den Interviews bleibt diese Gruppe weitestgehend unsichtbar, da die Genese von PAYL nur bezüglich des Interesses der Krankenversicherungen an PAYL abgefragt wurde. Zwar wird PAYL speziell von einigen Expert*innen als Trend gerahmt, zu dem es sich zu verhalten gelte, dessen Initiation wird aber nicht explizit Unternehmensberatungen zugerechnet. Auch wenn es bereits seit 2009 PAYL-artige Angebote (Vitality in Südafrika) gibt und auch wissenschaftliche Auseinandersetzungen mit dem Konzept PAYL als einem theoretisch möglichen Zukunftsszenario bestanden (Braun und Nürnberg 2015), wird der Begriff an sich erstmals im August 2015 virulent. Das hierfür ausschlaggebende Diskursereignis scheint nach unserer Literaturanalyse ein Konzeptpapier der Unternehmensberatung Ernst \& Young (2015) mit dem Titel: „Introducing ,Pay As You Live“ (PAYL) Insurance. Insurance that rewards a healthier lifestyle“ zu sein. Auch wenn die Wertschöpfung auf Kundenseite hier bereits in der Überschrift in Form eines gesünderen Lebensstils und Belohnung angesprochen wird, ist es vor allem die monetäre Wertschöpfung auf Unternehmensseite, die Dreh- und Angelpunkt der Veröffentlichung ist. Eine Schlüsselfrage an die Versicherungen sei dementsprechend: „How to create competitive advantage through leveraging digital technology?" Auch Beratungsfirmen wie Sopra Steria Consulting räsonieren über das ,Zeitalter des Self-Trackings“ und versprechen, dass ,Tracking-Tarife [...] die Möglichkeit [bieten], Kunden mit guten Risikoeigenschaften zu gewinnen“ (Schoss 2015).

Ganz im Sinne klassischer Geschäftsmodelle, welche die Dynamik zwischen Unternehmen und Kund*innen fokussieren, wird selbstvermessenden Akteuren aus Richtung der Unternehmensberatungen unterstellt, sie hätten ein vitales Interesse an PAYL-Tarifen: „Kunden verlangen zunehmend nach individuellen Versicherungsmodellen, die exakt auf ihre Lebenslage zugeschnitten sind“ (adesso AG 2015). Viele Menschen betreiben zwar in großem Stil Selbstvermessung, für das von den Unternehmensberatungen diagnostizierte Verlangen nach PAYL-Tarifen auf Versichertenseite als Initiator von PAYL-Geschäftsmodellen werden jedoch keine belastbaren Belege angeführt. 
Seit Ende 2015 hat der Diskurs um PAYL sowohl in medialen und wissenschaftlichen Resonanzräumen als auch im Bereich der Versicherungen selbst an Bedeutung gewonnen. Dass das Thema jedoch durch ein Beratungsunternehmen auf die Landkarte gesetzt wurde, lässt innehalten und ist unseres Erachtens auch für den weiteren Diskursverlauf bedeutsam. Dies zeigt sich auch in den Interviews: PAYL wird stark als ein von außen an die Versicherungen herangetragener Trend gerahmt, den es zu adressieren gelte. Die Entwicklung bzw. Implementierung von PAYL wird in diesem Zusammenhang oftmals als zwangsläufig konzipiert. Dies lässt sich zunächst als erfolgreiches Marketing der Unternehmensberatungen werten, untergräbt aber Fragen der Nachhaltigkeit sowie den Entscheidungscharakter gesundheitspolitischer Entwicklungen.

Die Entscheidung für oder gegen PAYL sollte genau das sein, eine Entscheidung als Abwägungsprozess, idealerweise auf der Grundlage des wissenschaftlich nachgewiesenen präventiven Nutzens oder des Fehlens eines solchen. Um sinnvolle Entscheidungen zu PAYL zu treffen, müssen diese also zunächst als Entscheidungen gerahmt werden. Dies erfordert ein tiefgreifendes Umdenken. Erste Ansätze dazu finden sich in der Technikfolgenabschätzung (TA), die das Denken in Alternativen als einen Öffnungsprozess propagiert, der mit der Frage ,Wie wollen wir leben?` beginnt (Dobroć et al. 2018). Im Hinblick auf PAYL sollte sie ergänzt werden um die Fragen, welche Formen der Prävention sinnvoll sind und welchen Preis wir bereit sind, dafür zu zahlen. Dafür ist ein klares Nutzenversprechen unabdingbar. PAYL nur als Trend zu verstehen, der uns aus unbelegten monetären Erwägungen zum Handeln zwinge, ist kontraproduktiv.

\subsection{Datensouveränität}

Ein zentrales Thema in der Auseinandersetzung mit PAYL ist der Datenschutz, daher wurde auch in den Interviews nach etwaigen Bedenken gefragt. Wenngleich einige interviewte Expert*innen die bestehenden Datenschutzregeln als eher innovationshemmend bezeichneten, besteht nahezu Konsens darüber, dass Datenschutz ein unverzichtbarer Wert sei. Über ihre Daten zu verfügen und sie im Falle von PAYL abzugeben, sei nach Dafürhalten der meisten interviewten Personen aber Sache des Einzelnen.

Datensouveränität, so verstanden als die Selbstbestimmung über persönliche Daten, wird von allen interviewten Stakeholdern ebenfalls als unbestritten wertvoll konzipiert: „Datenschutz und Datensicherheit genießen den höchsten Stellenwert“ (AOK Plus 2020). Allerdings wird die Bedeutung des Wertes Souveränität in den empirischen Daten mit dem Hinweis auf die Verantwortung und alleinige Entscheidung der Versicherten betont. Die Rolle der Versicherung hingegen sei nur die des Anbieters, ohne Daten zu verlangen oder zu speichern: „Der Patient in der digitalen Welt ist aufgeklärt und selbstbestimmt. Grundvoraussetzung ist dabei die Stärkung der Datensouveränität des Versicherten. Er hat als einziger den direkten Zugriff auf seine Daten. Er entscheidet, wem er, wie lange und wofür die Nutzung seiner Daten erlaubt. Der Versicherte ist und bleibt Herr seiner Daten“ (AOK Plus 2020).

Die Bedeutung des Wertes Datensouveränität ist aus Versichertensicht ein zweischneidiges Schwert: Zwar wird der Wert gestärkt, indem Versicherte auch rechtlich 
als Besitzer*innen ihrer Daten gedacht werden, jedoch wird den Versicherten dadurch auch ein Gros der Verantwortung für ihre Daten übertragen, ohne dass sie zu jeder Zeit angemessen über die Konsequenzen ihrer Entscheidungen informiert wären. Wie dargestellt, bieten die Krankenversicherungen keine technische Insellösung an, bei welcher der Datenfluss auf die Versicherten und die Versicherung beschränkt wäre. Dies erhöht die Anzahl an Stakeholdern mit jeweils eigenem Interesse an den Daten. Nichtsdestotrotz ist eine technische Lösung ohne sie nicht vorgesehen, sodass für eine Teilhabe am Bonusprogramm auch den Einschränkungen des eigenen persönlichen Datenschutzes für genutzte Plattformen zugestimmt werden muss.

Die Realisierbarkeit des Wertes Datensouveränität steht und fällt mit der Informiertheit der Versicherten. Da keine der Versicherungen in ihren Nutzungsbedingungen das komplette Daten-Ökosystem abbildet, kann nicht von der Informiertheit der Versicherten ausgegangen werden. Inwieweit eine solche Darstellung überhaupt möglich und logisch nachvollziehbar wäre, ist zudem fraglich. Die Einwilligung in die Nutzungsbedingungen (auch der notwendigen anderen Unternehmen) darf daher nicht mit informed consent (informierter Einwilligung) verwechselt werden. Janssen und Geitz gehen soweit zu sagen, dass die Datendistribution in den unterschiedlichen Informationsnetzwerken und Clouds längst so unüberschaubar geworden sei, dass eine ,technische Verunmöglichung des Nachvollzugs [...] den Rechtsanspruch auf Informiertheit [konterkariere]; dieser Anspruch ist gegenwärtig also eher als eine Rechtsfiktion aufzufassen, die weniger der Gesundheit des Patienten als der Auswertung seines Datenkörpers durch Dritte dient“" (Arnold 2020).

Wie kann es weitergehen? Wenn sich Krankenversicherungen dazu entscheiden, PAYL-Tarife oder -Bonusprogramme unter Berücksichtigung des Wertes Datensouveränität anzubieten, ist es ratsam, eigene Funktionen in deren Apps zu implementieren, welche die Endgeräte bzw. deren Sensoren direkt ansprechen. Hierdurch müssten sie zwar die Datensicherheit selbst gewährleisten, wären aber nicht auf externe Anbieter und deren Datenpolitik angewiesen. Selbst in diesem Falle bliebe der Weg der Daten hin zur Krankenversicherung lang und mit zahlreichen Risiken verbunden. Bislang sind die Versicherungen durch ihre Abhängigkeit von Plattformanbietern selbst nicht souverän. Zumindest auf der Ebene der Wearables aber gibt aus dem Umfeld von AOK Systems Überlegungen, eigene Angebote zu machen.

Da das bestehende Datenschutzrecht grundsätzlich über die Möglichkeit der Einwilligung auch der Erhebung intimster Daten keine Grenzen setzt, die Versicherungen aber in einem Abhängigkeitsverhältnis stehen und die umfassende Darstellung der Konsequenzen durch eine solche Einwilligung eine Fiktion bleiben muss, stimmen wir ebenso wie die Projektgruppe der Verbraucherschutzministerkonferenz (VSMK) der Empfehlung der Justizministerkonferenz zu, ,auf Grundlage von Art. 9 Abs. 4 DSGVO die Versicherten wenigstens vor einer laufenden Erfassung hochsensibler Gesundheitsdaten zum Zwecke der Prämiengestaltung zu schützen“ und ein gesetzliches Verbot durchzusetzen. „Darüber hinaus sieht die Projektgruppe die Notwendigkeit von daten-ethischen Leitlinien und Selbstbeschränkungen der Versicherungsunternehmen“ (VSMK 2019, S. 9). Es scheint sinnvoll, diese Vorschläge nicht nur auf PAYL-Tarife, sondern auch bereits auf PAYL-Bonusprogramme anzuwenden. 


\subsection{Evidenzproblem PAYL}

Gesetzlich besteht für die Krankenkassen in Deutschland nach § 1 SGB V der Auftrag, u. a. Prävention zu leisten. PAYL-Bonusprogramme werden von den interviewten Stakeholdern, die sich eher affirmativ zu PAYL äußern, als ein Instrument gesehen, diesem Präventionsauftrag zu entsprechen. Das Ziel Prävention hat eine wirtschaftliche Komponente nicht zuletzt deshalb, weil die Ausgestaltung von PAYL als Bonusprogramm rechtlich erfordert, dass die Aufwendungen sich mittelfristig selbst finanzieren. Gesundheitliche Prävention und wirtschaftlicher Nutzen sind also im Fall PAYL aufs engste verschränkt. Interviewte Proponent*innen und Opponent*innen von PAYL sind sich jedoch sowohl uneinig darüber, ob Prävention erreicht wird, als auch ob Kosten eingespart werden. PAYL hat bezüglich beider Wissenskonflikte ein Evidenzproblem: Sowohl für den Wert der Präventivwirkung als auch für den der Kostenersparnis, die gleichermaßen auf Versicherten- als auch Versicherungsseite als erstrebenswert betont werden, ist nicht ausreichend belegt, ob sie verwirklicht werden können. Nach Auffassung einiger interviewter Expert*innen sind diese Fragen schwierig bis unmöglich aufzulösen.

Dies scheint im besonderen Maße für die Möglichkeit von Prävention zu gelten. Zwar wird Gesundheitsförderung (neben dem finanziellen Einsparpotenzial) von Krankenversicherungen als der Hauptgrund für den PAYL-Ansatz artikuliert. Wie er sich aber zu anderen Präventionsansätzen verhält und ob ein Zusammenhang festgestellt werden kann, wurde bisher nicht nachgewiesen. Stakeholder wie die Verbraucherschutzministerkonferenz sowie der Sachverständigenrat für Verbraucherfragen sehen die Präventivwirkung nicht belegt (vgl. VSMK 2019; SVRV 2018). Interviewte Expert*innen mit wissenschaftlichem Hintergrund stellen aufgrund der komplexen Erhebungsbedingungen von Selbstvermessung sogar die empirische Realisierbarkeit der Untersuchung der Präventivwirkung in Frage.

Der Wunsch nach Verknüpfung von Selbstvermessung und Versicherungssystem wird auch dahingehend problematisch, wenn man die Kurzlebigkeit, welche die Praxis der Selbstvermessung mitunter begleitet, zugrunde legt. Piwek et al. verweisen in diesem Zusammenhang auf Ledger und McCaffrey (2014): „,recent surveys showed that $32 \%$ of users stop wearing these devices after six months, and $50 \%$ after one year. Many wearables suffer from being a solution in search of a problem “ "(Piwek et al. 2016). Es gibt in den Interviews mit Anwender*innen auch erste Anzeichen dafür, dass die intrinsische Motivation, sich zu bewegen, durch PAYL nicht nur zusondern sogar abnehmen könnte.

Die Realisierung des Wertes Kostenersparnis wirkt zumindest auf Kund*innenseite realistisch und die Teilnahme an PAYL-Bonusprogrammen wird von den interviewten Anwender*innen durchweg als lohnenswert beschrieben. Die Werte Gesundheit und Kosteneinsparung sind im Fall von PAYL-Bonusprogrammen auch rechtlich miteinander verzahnt: Nach Paragraph 65a Absatz 3 SGB V sind die Krankenkassen verpflichtet, den wirtschaftlichen Erfolg von Präventionsmaßnahmen nachzuweisen, um aus diesen Einsparungen Boni finanzieren zu dürfen. Man könnte davon ausgehen, dass eine Entsprechung des Wertes Kostenersparnis auch bedeute, die Maßnahmen seien präventiv wirksam. Dies ist, wie erwähnt, bisher nicht belegt und Präventionswirkung kann auch auf andere Gründe zurückgeführt 
werden. Allen voran besteht die Möglichkeit, dass die Maßnahmen bereits Kunden mit einer positiven Risikoselektion ansprechen, also bereits sportlich aktive, junge und sozio-ökonomisch besser gestellte Menschen (vgl. Arentz und Rehm 2016, S. 6f.). Zudem wurde im Interview mit einem Stakeholder aus der gesetzlichen Krankenversicherung bestätigt, dass die rechtlich vorgesehene Evaluation die gesamten Präventionsprogramme einer Krankenkasse gebündelt evaluieren kann, ohne PAYL gesondert zu betrachten.

Eine interviewte Versicherungsmathematikerin der Deutschen Aktuarvereinigung sieht im Bereich der Tarife das Problem, dass man mindestens 20 Jahre alte Daten bräuchte, um die finanzielle Sinnhaftigkeit von PAYL beurteilen zu können - eine Herausforderung für die übereilte Implikation technologischer Systeme. Selbst die Hoffnung, dass trotz der mangelnden Evaluationsmöglichkeiten faktische Kostenersparnis realisiert werden kann, ergibt sich für die Versicherungen aus der Bindung von Versicherten mit positiver Risikoselektion. Als first mover besteht die Chance, diesen Vorteil gegenüber anderen Versicherungen auszubauen. Doch auch wenn dies kurzfristige Profite zeitigen sollte, geben Arentz und Rehm zu bedenken, dass es längerfristig anders aussehen könnte: Durch kurzfristige Ersparnis könnte den Versicherungen langfristig der Wert der Kostenersparnis bedroht sein: „,[E]ven a certain behavior that positively causally affects one's health and thus decreases short-term health care expenditure might not reduce one's lifetime health care expenditure. If individuals live shorter, they are less likely to develop severe diseases that adjusting one's behavior cannot help to avoid. The cost of treating these diseases in later stages of life might exceed the cost of treating those diseases correlated to an unhealthy lifestyle. Empirically, this has been shown for smoking (Barendregt et al. 1997) and overweight (van Baal et al. 2008) for instance. In that case, the insurance system as a whole would, profit" from unhealthy habits because they would lead to less costs for the system in the long run“ (Arentz und Rehm 2016, S. 5). Den normativen Horizont dieser Annahmen außer Acht lassend könnte zynisch betrachtet der Wert Kostenersparnis langfristig für das Gesundheitssystem und letztendlich auch das Rentensystem also gefährdet sein.

\section{Zusammenfassung und Ausblick}

In diesem Beitrag haben wir die Definition eines PAYL-Systems, den aktuellen Stand der Technik und eine empirische Untersuchung der möglichen Konflikte eines solchen Systems in Bezug auf Krankenversicherungsmitglieder, Krankenversicherungsunternehmen und die Gesellschaft vorgestellt. Wir haben drei Hauptkonflikte identifiziert, die bei der Gestaltung und Implementierung solcher Systeme berücksichtigt werden sollten. Erstens rahmen die Versicherungsunternehmen PAYL als Trend, zu dem es sich zu verhalten gelte, und implementieren daher übereilt Systeme, die möglicherweise weder ausgereift noch gesellschaftlich wünschenswert sind. Ein stärkerer Fokus gerade auf die Konzeption von PAYL als Entscheidung statt ökonomisch forcierter Zwangsläufigkeit wäre in diesem Zusammenhang notwendig. Zweitens existiert PAYL als Teil eines Daten-Ökosystems, PAYL-Systeme sind technisch also als System im System ausgestaltet. Je nachdem, wo die Versicherungs- 
unternehmen die Grenzen ihrer Systeme ziehen, verlagern sie die Verantwortung in Bezug auf Datenschutz und Datensicherheit auf das Individuum und suggerieren kontrafaktisch dessen Souveränität bei faktischer Überkomplexität und mangelnder Nachvollziehbarkeit der Konsequenzen. Schließlich ist die Behauptung, PAYL sei ein Instrument zur Prävention, das zu Einsparungen im Gesundheitswesen führen soll, nicht bewiesen und ggf. nicht beweisbar. Die medizinische und wirtschaftliche Evidenz von PAYL sollte von einem unabhängigen Gremium untersucht und mit anderen Präventionsmaßnahmen abgeglichen werden. Einige interviewte Expert*innen favorisieren umfassendere staatliche Präventionsangebote, wie sie in anderen Ländern üblich sind, gegenüber kleinteiligen und versicherungsgetriebenen Angeboten. Besonders hier scheint ein Vergleich sinnvoll.

Funding Open Access funding enabled and organized by Projekt DEAL.

Open Access Dieser Artikel wird unter der Creative Commons Namensnennung 4.0 International Lizenz veröffentlicht, welche die Nutzung, Vervielfältigung, Bearbeitung, Verbreitung und Wiedergabe in jeglichem Medium und Format erlaubt, sofern Sie den/die ursprünglichen Autor(en) und die Quelle ordnungsgemäß nennen, einen Link zur Creative Commons Lizenz beifügen und angeben, ob Änderungen vorgenommen wurden.

Die in diesem Artikel enthaltenen Bilder und sonstiges Drittmaterial unterliegen ebenfalls der genannten Creative Commons Lizenz, sofern sich aus der Abbildungslegende nichts anderes ergibt. Sofern das betreffende Material nicht unter der genannten Creative Commons Lizenz steht und die betreffende Handlung nicht nach gesetzlichen Vorschriften erlaubt ist, ist für die oben aufgeführten Weiterverwendungen des Materials die Einwilligung des jeweiligen Rechteinhabers einzuholen.

Weitere Details zur Lizenz entnehmen Sie bitte der Lizenzinformation auf http://creativecommons.org/ licenses/by/4.0/deed.de.

\section{Literatur}

adesso AG (2015) Geschäftsmodelle 4.0 - Was die Assekuranz von anderen Branchen lernen kann: Studie der Versicherungsforen Leipzig GmbH im Auftrag der adesso AG, Dortmund, Leipzig. https://www. adesso.de/adesso/adesso-de/branchen/versicherungen/sonderthemen/ergebnisbroschuere-studiegeschaeftsmodelle40-web.pdf. Zugegriffen: 28. Febr. 2021

AOK Plus (2020) Positionen und Statements, Position Datenschutz. https:/www.aok.de/pk/plus/inhalt/ digitalisierun\%20g-im-gesundheitswesen/. Zugegriffen: 28. Febr. 2021

Arentz C, Rehm R (2016) Behavior-based tariffs in health insurance: compatibility with the German system. Otto-Wolff-discussion paperUR. https://www.econstor.eu/handle/10419/201594. Zugegriffen: 28. Febr. 2021

Arnold F (2020) Tagungsbericht zur Tagung „Künstliche Intelligenz und Weltverstehen“ des Leopoldina-Zentrums für Wissenschaftsforschung in Kooperation mit dem Interdisciplinary Network for Studies Investigating Science and Technology (INSIST), Halle (Saale). https://www.hsozkult.de/ conferencereport/id/tagungsberichte-8826. Zugegriffen: 28. Febr. 2021

van Baal PHM, Polder JJ, de Wit GA, Hoogenveen RT, Feenstra TL, Boshuizen HC, Engelfriet PM, Brouwer WBF (2008) Lifetime medical costs of obesity: prevention no cure for increasing health expenditure. PLoS Med 5:e29. https://doi.org/10.1371/journal.pmed.0050029

Barendregt JJ, Bonneux L, van der Maas PJ (1997) The health care costs of smoking. New Engl J Med 337:1052-1057. https://doi.org/10.1056/NEJM199710093371506

BCG - Boston Consulting Group (2017) GKV 4.0-Trend-Monitor. https://image-src.bcg.com/Images/ BCG_GKV4-0_tcm9-178444.pdf. Zugegriffen: 28. Febr. 2021

Braun M, Nürnberg V (2015) Verhaltensbasierte Versicherungstarife - innovative E-Health-Initiative oder Ausstieg aus der Solidargemeinschaft? G S Gesundh Sozialpolitik 69:70-75. https://doi.org/10.5771/ 1611-5821-2015-1-70 
Clarke AE (2005) Situational analysis: grounded theory after the postmodern turn. SAGE, Thousand Oaks

Corbin JM, Strauss AL (2015) Basics of qualitative research: techniques and procedures for developing grounded theory. SAGE, Los Angeles

Dahrendorf R (1961) Elemente einer Theorie des sozialen Konflikts. In: Dahrendorf R (Hrsg) Gesellschaft und Freiheit. Zur soziologischen Analyse der Gegenwart. Pieper, München, S 197-235

Dobroć P, Krings B-J, Schneider C, Wulf N (2018) Alternativen als Programm: Plädoyer für einen Perspektivenwechsel in der Technikfolgenabschätzung. TATuP 27:28-33. https://doi.org/10.14512/tatup.27. 1.28

Ernst \& Young (2015) Introducing 'Pay As You Live' (PAYL) Insurance. Insurance that rewards a healthier lifestyle. https://nanopdf.com/download/introducing-pay-as-you-live-payl-insurance_pdf. Zugegriffen: 28. Febr. 2021

Evers-Wölk M, Oertel B, Sonk M (2018) Gesundheits-Apps - Innovationsanalyse: TAB-Arbeitsbericht Nr. 179. http://www.tab-beim-bundestag.de/de/untersuchungen/u30000.html. Zugegriffen: 28. Febr. 2021

Kruse Brandão T, Wolfram G (2018) Digital Connection: Die bessere Customer Journey mit smarten Technologien - Strategie und Praxisbeispiele. Springer, Wiesbaden

Ledger D, McCaffrey D (2014) Inside wearables: how the science of human behavior change offers the secret to long-term engagement. http://pop.h-cdn.co/assets/cm/15/13/5512f7e752a4e_-_Wearablesand-the-Science-of-Human-Behavior-Change-EP4.pdf. Zugegriffen: 28. Febr. 2021

MSC - Mücke Sturm Company (2018) Studie: Bereitstellung digitaler Services durch die Krankenkassen in Deutschland. https://www.muecke-roth.de/wp-content/uploads/Praesentation-Studienergebnisse20180630-Final.pdf. Zugegriffen: 28. Febr. 2021

Piwek L, Ellis DA, Andrews S, Joinson A (2016) The rise of consumer health wearables: promises and barriers. PLoS Med 13(2). https://doi.org/10.1371/journal.pmed.1001953

Schoss M (2015) Zukunft von Tracking-Tarifen: Umfrage zur Nutzerakzeptanz von PAYL. Sopra Steria Consulting,

SVRV - Sachverständigenrat für Verbraucherfragen (2018) Gutachten Verbrauchergerechtes Scoring. https://www.svr-verbraucherfragen.de/dokumente/verbrauchergerechtes-scoring/. Zugegriffen: 28. Febr. 2021

VSMK - Verbraucherschutzministerkonferenz (2019) Telematiktarife im Versicherungsbereich: Abschlussbericht der Projektgruppe der Arbeitsgemeinschaft Wirtschaftlicher Verbraucherschutz. https://www.verbraucherschutzministerkonferenz.de/documents/anlage-1_1559131158.pdf. Zugegriffen: 28. Febr. 2021

Wiener M, Saunders C, Marabelli M (2020) Big-data business models: a critical literature review and multiperspective research framework. J Inf Technol 35:66-91 